\title{
Theoretical and Numerical Study of Seismoelectric Logs for Logging While Drilling (LWD)
}

\author{
Xiang-Yang SUN ${ }^{1, a}$, Yong-Peng ZHAO ${ }^{2}$ \\ ${ }^{1}$ High tech Zone(West), Chengdu, Sichuan, China Xi yuan Avenue, No. 2006 , University of Electronic Science and \\ technology
}

\begin{abstract}
When the acoustic field propagates in the porous medium, the electromagnetic wave can be induced by the seismoelectric effects which can be used in the logging. This paper studies the seismoelectric wave field and deduces the formulations of the acoustic and electromagnetic field of the liquid in the well, and gives the acoustic and electromagnetic boundary conditions between the driller collar and the log liquid, when the driller collar is added. We also do the numerical simulation of the seismoelectric wave field excited by monopole source, study the velocity dispersion characteristics of the acoustic and electromagnetic field, and investigate the detective depth of the seismoelectric logs. Through the simulation we know the seismoelectric logs can avoid the interference of drill collar wave. This paper also provides theoretical guidance for the design of receiving antenna.
\end{abstract}

\section{Introduction}

With the development of the world economy, the shortage of oil and gas has become the main restricting factor of economic and social development[1].And the oil and gas are mainly stored in the porous media[2].So the application of seismoelectric effects in the exploration of oil and gas is very promising, especially in the search of "remaining oil"[3]. Seismoelectric effects refer to the excitation of electromagnetic wave excited by the seismic wave in the porous medium, It is a process of energy conversion between mechanical energy and electromagnetic energy in nature. Seismoelectric logs have better recognitive ability of oil and gas, and better detective ability than pure electric logging and seismic logging. Seismoelectric logs are also sensitive to the variation of formation parameters, so we can get more information.

A simplified method for Seismoelectric logs is proposed by Hu Hengshan and Liu Jiaqi, which ignores the influence of electromagnetic field and solve the acoustic field independently first, and then solve the electromagnetic field by using seismic and electric coupling relationship[4,5]. Guan Wei and $\mathrm{Hu}$ hengshan introduces the Maxwell equations with the percolation current as the current source, and uses the Hertz vector to represent the solutions[6]. Jin Zhiwen derived the expressions of the acoustic and the electromagnetic field in the porous medium excited by the multipole source[7].

Although there are a large number of theoretical researches about seismoelectric logs, but almost none considers the influence of drilling equipment such as the drill collar. Based on the above background, this paper studies the seismoelectric logs while drilling. And we give the analytical

\footnotetext{
${ }^{\text {a }}$ Corresponding author: sunxiangyang@uestc.edu.cn
} 
solution of the seismoelectric field and study the propagating characteristics of the acoustic and the electromagnetic field. We also obtain the velocity dispersion characteristics of the seismoelectric field, and investigate the detective depth of the seismoelectric logs.

\section{Formulation}

\subsection{Acoustic field}

In the simulation, we use the LWD's model as shown in the Figure 1, with radial stratification, followed by mud liquid layer, drill tool layer, mud liquid layer in the borehole and porous medium.

When add the drill collar, there will be a cylindrical outward displacement potential function for the acoustic wave in the borehole. Excited by multipole source, the formal solution is

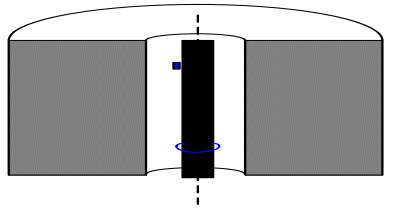

Figure1. model for logging while drilling(LWD)

$$
\varphi(r, \theta, k, \omega)=\sum_{n=0}^{\infty} \varphi_{n}(r, \theta, k, \omega)=\sum_{n=0}^{\infty}\left[A_{n}^{f} I_{n}(\eta r)+B_{n}^{f} K_{n}(\eta r)\right] \cos n \theta
$$

$\mathrm{k}$ is the axial wavenumber, $\eta=\sqrt{l^{2}-(\omega / V)^{2}}$ is the radial imaginary wavenumber. The displacement and sound pressure can be calculated according to the relationship between the displacement (or the pressure)and the potential function in cylindrical coordinates in the flood.

$$
\begin{gathered}
u_{r}^{o}(r, \theta, k, \omega)=\sum_{n=0}^{\infty}\left\{A_{n}^{o}\left[\frac{n}{r} I_{n}(\eta r)+\eta I_{n+1}(\eta r)\right]+B_{n}^{o}\left[\frac{n}{r} K_{n}(\eta r)-\eta K_{n+1}(\eta r)\right]\right\} \cos n \theta(2) \\
P_{f}^{o}(r, \theta, k, \omega)=\sum_{n=0}^{\infty} \rho_{f} \omega^{2}\left[A_{n}^{o} I_{n}(\eta r)+B_{n}^{o} K_{n}(\eta r)\right] \cos n \theta
\end{gathered}
$$

With no source inside the drill collar cavity, there is only inward wave field.

$$
\begin{aligned}
& u_{r}^{i}(r, \theta, k, \omega)=\sum_{n=0}^{\infty} A_{n}^{i}\left[\frac{n}{r} I_{n}(\eta r)+\eta I_{n+1}(\eta r)\right] \cos n \theta \\
& P_{f}^{i}(r, \theta, k, \omega)=\sum_{n=0}^{\infty} \rho_{f} \omega^{2} A_{n}^{i} I_{n}(\eta r) \cos n \theta
\end{aligned}
$$

The wave equations for the displacement potential function of the acoustic field in the tool layer are: $\nabla^{2} \varphi+k_{p}^{2} \varphi=0 \quad \nabla^{2} \chi+k_{S H}^{2} \chi=0 \quad \nabla^{2} \Gamma+k_{S V}^{2} \Gamma=0$

Where $k_{p}^{2}=\omega^{2} / c_{p}^{2}, k_{S H}^{2}=k_{S V}^{2}=\omega^{2} / c_{s}^{2}, c_{p}=\sqrt{(\lambda+2 \mu) / p}, c_{s}=\sqrt{\mu / p}$. and $\varphi$ is the displacement potential function of the longitudinal wave in the tool layer, $\chi$ denotes the displacement potential function of the SH wave, $\boldsymbol{\Gamma}$ is the displacement potential function of the SV wave. The solutions of the three potential functions are: 


$$
\begin{aligned}
& \varphi(r, \theta, k, \omega)=\sum_{n=0}^{\infty} \varphi_{n}=\sum_{n=0}^{\infty}\left[A_{n} I_{n}\left(\eta_{p} r\right)+B_{n} K_{n}\left(\eta_{p} r\right)\right] \cos n \theta \\
& \chi(r, \theta, k, \omega)=\sum_{n=0}^{\infty} \chi_{n}=\sum_{n=0}^{\infty}\left[C_{n} I_{n}\left(\eta_{s} r\right)+D_{n} K_{n}\left(\eta_{s} r\right)\right] \cos n \theta \\
& \Gamma(r, \theta, k, \omega)=\sum_{n=0}^{\infty} \Gamma_{n}=\sum_{n=0}^{\infty}\left[E_{n} I_{n}\left(\eta_{s} r\right)+F_{n} K_{n}\left(\eta_{s} r\right)\right] \cos n \theta
\end{aligned}
$$

We can obtain the displacement and stress of the acoustic field in the tool layer according to the relationship between the displacement and the potential function and the stress-strain's relationship in the elastic solid in the cylindrical coordinate[7].

In the porous medium, the displacement potential function of each body wave satisfies the following equations:

$$
\nabla^{2} \varphi_{l}+k_{p}^{2} \varphi_{l}=0 ; \nabla^{2} \chi+k_{S H}^{2} \chi=0 ; \nabla^{2} \Gamma+k_{S V}^{2} \Gamma=0
$$

Where $\varphi_{l}$ is the displacement potential function of the longitudinal wave including fast longitudinal wave and slow longitudinal wave, $\chi$ denotes the displacement potential function of the SH wave, $\Gamma$ is the displacement potential function of the SV wave [6].

\subsection{Electromagnetic field}

There is a certain proportional relationship between the solid displacement and the seepage displacement of each body wave in the porous medium.

$$
w=\alpha_{p f} \nabla \phi_{f}+\alpha_{p s} \nabla \phi_{s}+\alpha_{s} \nabla \times(\chi \vec{z})+\alpha_{s} \nabla \times \nabla \times(\Gamma \vec{z})
$$

So the current flow can be calculated as

$$
J_{s}=\nabla \psi_{1}+\nabla \psi_{2}+\nabla \times F_{1}+\nabla \times \nabla \times F_{2}
$$

According to the electromagnetic theory, when the excitation source is seepage current, we can get the equations of the Hertz vector as following:

$$
\begin{aligned}
& \left(\nabla^{2}-\mu \bar{\varepsilon} \frac{\partial^{2}}{\partial t^{2}}\right) \vec{\Pi}^{m}=-\overrightarrow{F_{1}}-\nabla \times \overrightarrow{F_{2}} \\
& \left(\nabla^{2}-\mu \bar{\varepsilon} \frac{\partial^{2}}{\partial t^{2}}\right) \vec{\Pi}^{e}=-\frac{\nabla \psi_{1}+\nabla \psi_{2}}{i \omega \varepsilon}
\end{aligned}
$$

When the source is individually $\nabla \psi_{1}, \nabla \psi_{2},-\overrightarrow{F_{1}},-\nabla \times \overrightarrow{F_{2}}$, one obtains the particular solutions:

$$
\vec{\Pi}_{1}^{e}=\frac{\nabla \psi_{1}}{i \omega \varepsilon\left(k_{e m}^{2}-k_{p f}^{2}\right)}, \vec{\Pi}_{2}^{e}=\frac{\nabla \psi_{2}}{i \omega \varepsilon\left(k_{e m}^{2}-k_{p s}^{2}\right)}, \vec{\Pi}_{3}^{m}=-\frac{\vec{F}_{1}}{k_{e m}^{2}-k_{p s}^{2}}, \vec{\Pi}_{4}^{m}=-\frac{\nabla \times \vec{F}_{2}}{k_{e m}^{2}-k_{p s}^{2}}
$$

Then get the solution of equations in the passive situation: 


$$
\vec{\Pi}_{0}^{e}=R_{e} \cdot A_{e} \cdot K_{n}\left(\eta_{e m 1} r\right) \quad \vec{\Pi}_{0}^{m}=R_{e} \cdot A_{m} \cdot K_{n}\left(\eta_{e m 1} r\right)
$$

According to the Hertz vector above all, we can calculate the electromagnetic field in the porous formation .

$$
\begin{aligned}
& \vec{E}=\vec{E}_{1}+\vec{E}_{2}+\vec{E}_{3}+\vec{E}_{4}+\vec{E}_{0} \\
& \vec{H}=\vec{H}_{1}+\vec{H}_{2}+\vec{H}_{3}+\vec{H}_{4}+\vec{H}_{0}
\end{aligned}
$$

There is electromagnetic field in the borehole which is not excited by the acoustic field but caused by the boundary conditions. The Hertz electric potential and Hertz magnetic potential in the borehole meet Helmholtz equation, respectively. When the drill collar added, there will be a cylindrical outward function for Hertz electric potential and Hertz magnetic potential in the borehole, individually:

$$
\vec{\Pi}_{0}^{e}=R_{e} \bullet B_{e} \bullet I_{n}\left(\eta_{e m 1} r\right)+R_{e} \bullet C_{e} \bullet K_{n}\left(\eta_{e m 1} r\right) \quad \vec{\Pi}_{0}^{m}=R_{e} \cdot B_{m} \cdot I_{n}\left(\eta_{e m 1} r\right)+R_{e} \cdot C_{m} \cdot K_{n}\left(\eta_{e m 1} r\right)
$$

Then we can obtain the electromagnetic field according to the relationship between the field and the Hertz potential.

\subsection{Boundary condition}

In the formal solutions of the acoustic and the electromagnetic field, there are unknown coefficients. These coefficients can be obtained by the boundary conditions of the acoustic and the electromagnetic field on the interface of different media.

The boundary conditions of acoustic field at the interface between drill collar and fluid inside or outside the drill collar satisfy:

Inner fluid

Continuity of normal stress : $u_{r}^{i}=u_{r}$

Continuity of normal stress : $-P_{f}^{i}=\tau_{r r}$

Continuity of tangential stress :0 $=\tau_{r z}$

Continuity of tangential stress : $0=\tau_{r \theta}$

\section{Outer fluid}

Continuity of normal stress : $u_{r}^{o}+v_{1}^{s}=u_{r}$

Continuity of normal stress : $-\left(P_{f}^{o}+\sigma_{1}^{s}\right)=\tau_{r r}$

Continuity of tangential stress : $0=\tau_{r z}$

Continuity of tangential stress : $0=\tau_{r \theta}$

The boundary conditions of acoustic field at the interface between drill collar and fluid inside or outside the drill collar satisfy:

Continuity of fluid flow : $u_{r}^{o}+v_{2}^{s}=u_{r}^{1}+w_{r}^{1}$

Continuity of Normal stress : $-\left(P_{f}^{o}+\sigma_{2}^{s}\right)=\tau_{r r}^{1}$

Continuity of Tangential stress : $0=\tau_{r z}^{1}$

Continuity of Tangential stress : $0=\tau_{r \theta}^{1}$

Continuity of fluid pressure $: P_{f}^{o}+\sigma_{2}^{s}=P^{1}$

$\mathrm{i}$ and o refer to the fluid medium inside and outside the drill collar, Respectively. 1 represent the porous medium. The matrix of acoustic boundary condition is

$$
\left[m_{i j}\right]_{13 \times 13} \bullet\left\{a_{j}\right\}_{13 \times 1}=\left\{b_{j}\right\}_{13 \times 1}
$$


Where $a_{j}$ is the coefficient of the displacement potential function, $b_{j}$ is the devotion of the source. The tangential components of electric field and magnetic field continue at the wall of the well, so we can obtain the equations:

$$
E_{\theta A}=E_{\theta B}, \quad E_{z A}=E_{z B}
$$

The tangential components of electric field equal to 0 at the interface between the drill collar and the fluid outside the drill collar.

$$
H_{\theta A}=H_{\theta B}, \quad H_{z A}=H_{z B}
$$

The matrix of boundary condition of electromagnetic field is

$$
\left[m_{i j}\right]_{6 \times 6} \bullet\left\{a_{j}\right\}_{6 \times 1}=\left\{b_{j}\right\}_{6 \times 1}
$$

Where $a_{j}$ is the coefficient of Hertz vector potential. $b_{j}$ is the the electromagnetic wave induced by the acoustic field in the porous medium.

\section{Numerical simulation}

The source is a monopole source with Richer wavelet as excitation function, and the center frequency is $7 \mathrm{kHz}$, the receiving points are located at the wall of the well. The starting position is $\mathrm{z}=1.3713 \mathrm{~m}$, and the distance between each receiving point is $0.1524 \mathrm{~m}$, there are seven receiving points. The full waveform of the seismoelectric wave field is as Figure2.

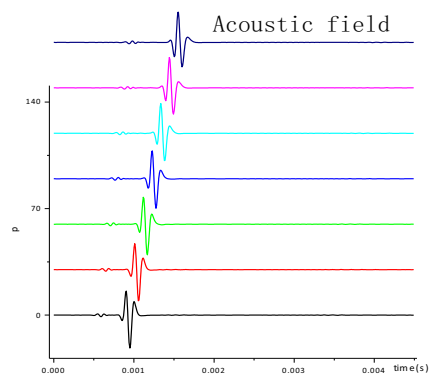

Figure 2a. Acoustic field

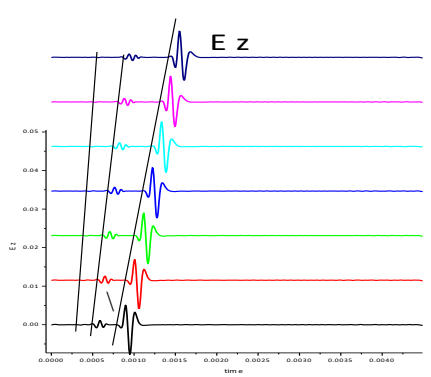

Figure 2c. Tangential electric field

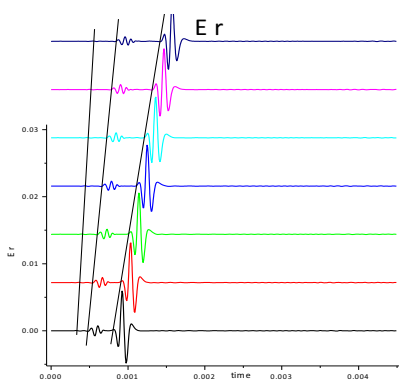

Figure 2b. Normal electric field

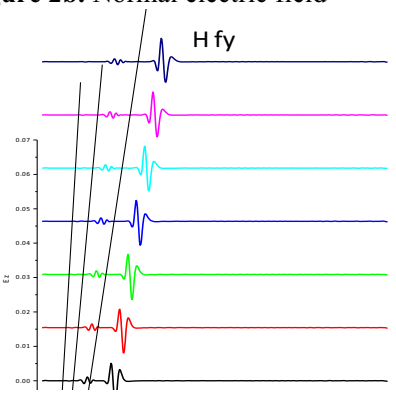

Figure 2d. Circumferential magnetic field

The three diagonal lines respectively go through the electromagnetic field coupled with fast longitudinal wave, transverse wave, Stoneley wave in Figure $2 \mathrm{~b}$, c, d. Their slopes indicate the acoustic wave velocity. We can see form the figure that the coupled electromagnetic field can reflect 
some properties of the acoustic field. The ratio of longitudinal wave and Stoneley wave or the ratio of shear wave and Stoneley wave of the acoustic field is less than that of the electromagnetic field.

We derive the velocity dispersion figures of the acoustic and the electromagnetic field by using multiple filter analysis method (MFT). We can see from Figure 3, when excited by monopole source, there is stronger drill collar interference for the acoustic field and less drill collar interference for the electromagnetic field.

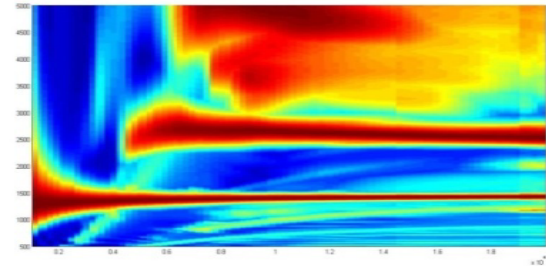

Figure 3a.Velocity dispersion of acoustic field

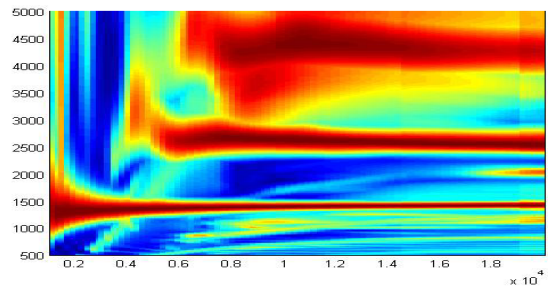

Figure 3c.Velocity dispersion of tangential electric field

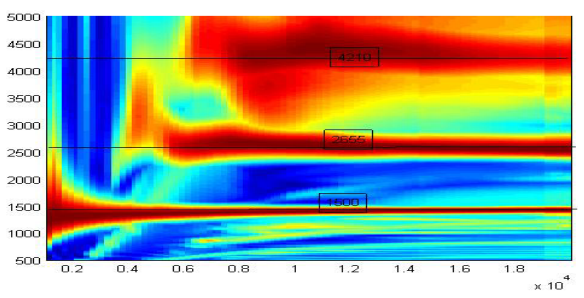

Figure 3b.Velocity dispersion of normal electric field

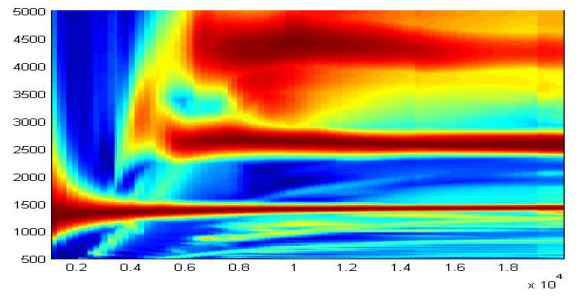

Figure 3d.Velocity dispersion of circumferential magnetic field

Figure $4 \mathrm{a}$ and Figure $4 \mathrm{~b}$ respectively compare the acoustic field, electric field and magnetic field. The amplitude of the axial component of the electric field is about 3 orders of magnitude smaller than that of the acoustic field, while their phases are almost the same. The amplitude of the radial component of the electric field is almost

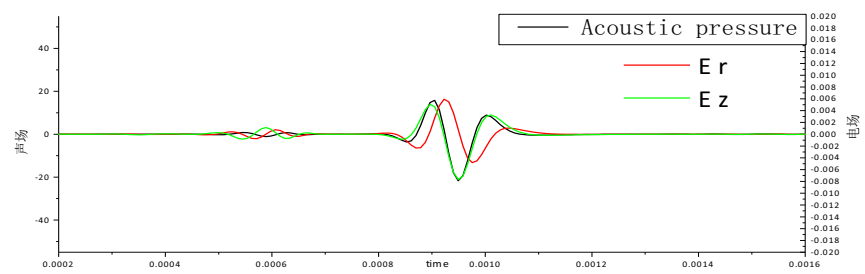

Figure4a. Contrast between electric field and sound field

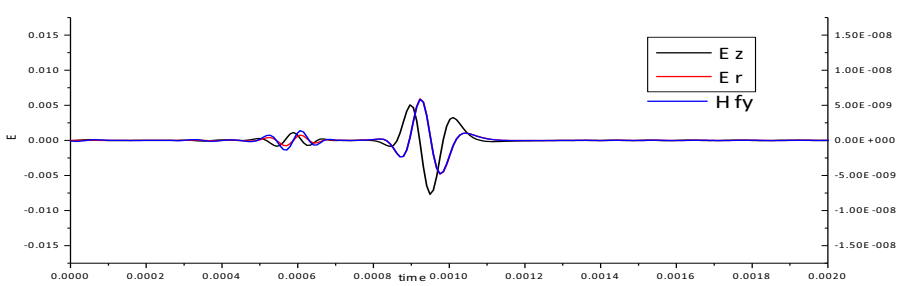

Figure 4b.Contrast between electric field and magnetic field 
Now we study the detective depth of the seismoelectric logs. The model is shown in Figure 5a. The current signal excited by the acoustic signal is distributed in all regions of the porous medium, as shown in the red part of the Figure $5 \mathrm{~b}$. In order to investigate how far the electrical signals that can be received by the electric current, we assume the current signal is only distributed in a part of the formation, as shown in the red part of the Figure 5c. The thickness of the layer that does not excite the current is $t$. Investigate the change of the received signal, When $t$ is changed.

Figure 6a investigates the change of the electric field the $t$ value, and Figure $6 \mathrm{~b}$ investigates the change of the amplitude of the electric field with $t$. As can be seen from the figure, the detective depth of seismoelectric logs is very shallow, there is one order of magnitude attenuation per $4 \mathrm{~cm}$, so the detective depth is about $2-3 \mathrm{~cm}$. At the same time, it is shown that the seismoelectric signal is mainly caused by the electric current of the electric double layer in the shallow stratum.

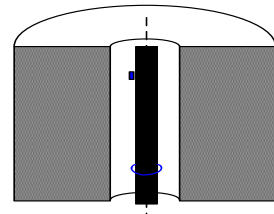

Figure 5a

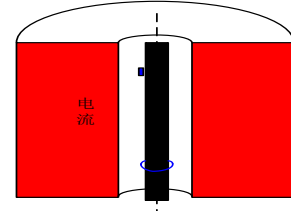

Figure 5b

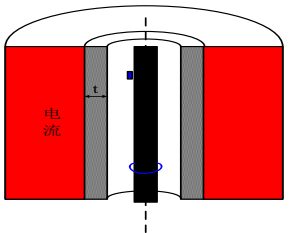

Figure 5c

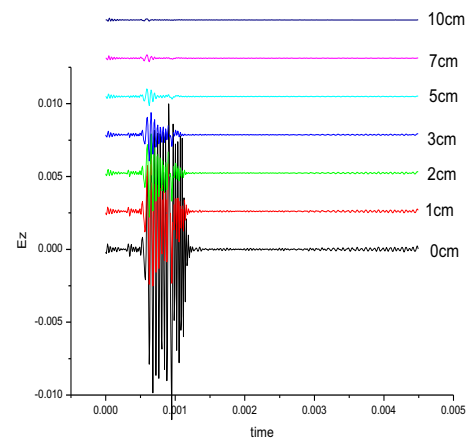

Figure 6a.The variation of axial electric field with $\mathrm{t}$

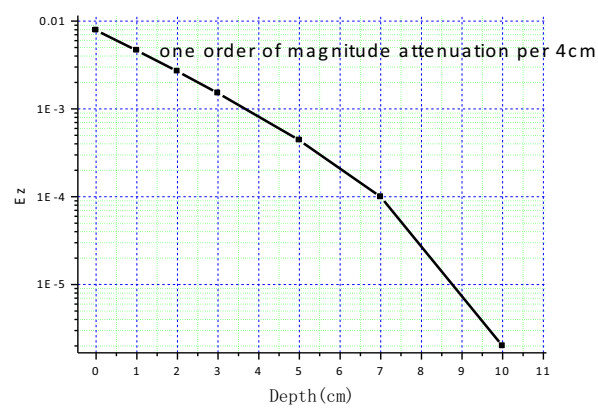

Figure 6b.The variation of axial electric field 's amplitude

\section{Summary}

Summarize the whole text, we can obtain the conclusions:

(1)Seismoelectric logs for LWD is feasible, the received signal can reflect acoustic wave velocity, which can be indirectly used in acoustic logging. It can be the response of P-wave, S-wave propagating along the formation and the Stoneley wave propagating along the borehole.

(2)Seismoelectric logs for LWD acoustic logging have certain advantages over acoustic logging: there is no collar wave interference for electromagnetic field when excited by monopole source.

(3)The simulation above, provides a reference for the design of the receiving antenna: There are 3 components in the electromagnetic field, namely, the axial electric field, the radial electric field and the circumferential magnetic field. We'd better receive the axial electric field.

(4)The receiving antenna can only receive the electric signal generated by the electric double layer of the shallow stratum, which is about $2 \sim 4 \mathrm{~cm}$.It is shown that the seismic signal is not generated by the interface between the wall and mud. At the same time, we can see that the electromagnetic field can reflect the characteristics of the acoustic field. 


\section{References}

1. Guan Wei. Study on wave field simulation of coupling effect of elastic wave and electromagnetic field in porous media [D]. Journal of Harbin Institute of Technology, 2009

2. Liu Hong, Li Youming. Some views on the exploration of oil and gas water by using seismic effect [J]. petroleum geophysical exploration, 1994,02:94-101.

3. Zhang Yan. The refraction of plane wave on the interface between fluid and porous media under the effect of electric shock [D]. Jilin University, 2011

4. Hu Hengshan, Liu Jiaqi, Yan Yan, Yan. Comparative analysis of the wave equations of and Pride, [J].,, 2003,01:107-112.

5. Hu Hengshan, Liu Jiaqi, Wang, Wang, et al. Based on the simplified Pride theory to simulate the logging response of electro acoustic effect [J]. Journal of Geophysics, 2003,02:259-264.

6. Guan Wei, Hu Hengshan, Chu zhaotan. Hertz vector acoustic induced electromagnetic field representation and multipole acoustic logging simulation [J]. Journal of physics, 2006,01:267-274.

7. Cui Zhiwen. Theoretical and numerical study of acoustic model of porous media and multipole source acoustic logging and multipole logging while drilling. Doctoral dissertation of Jilin University. 2004:52-8 